This is the peer reviewed version of the following article:

Nagar B., Dubal D.P., Pires L., Merkoçi A., Gómez-Romero P.. Design and Fabrication of Printed Paper-Based Hybrid Micro-Supercapacitor by using Graphene and Redox-Active Electrolyte. ChemSusChem, (2018). 11. : 1849 - . 10.1002/cssc. 201800426,

which has been published in final form at https://dx.doi.org/10.1002/cssc.201800426. This article may be used for non-commercial purposes in accordance with Wiley Terms and Conditions for Use of Self-Archived Versions. 
Full Paper

\section{Design and Fabrication of Printed Paper-Based Hybrid Micro- Supercapacitor by using Graphene and Redox-Active Electrolyte}

Bhawna Nagar, ${ }^{[\mathrm{a}, \mathrm{b}]}$ Dr. Deepak ${ }^{\wedge \wedge}$ P. Dubal 0000-0002-2337-676X, ${ }^{[\mathrm{a}, \mathrm{c}]}$ Dr. Luis Pires, ${ }^{[\mathrm{b}]}$ Prof. Arben Merkoçi, ${ }^{[\mathrm{b}, \mathrm{d}]}$ and Prof. Pedro Gómez-Romero*[a]

[a] <orgDiv/>Novel Energy-Oriented Materials Group, <orgName/>Catalan Institute of Nanoscience and Nanotechnology (ICN2), CSIC and The Barcelona Institute of Science and Technology

<street/>Campus UAB, <postCode/>08193<countryPart/>Bellaterra, <city/>Barcelona $(<$ country/>Spain $)$

E-mail:pedro.gomez@icn2.cat

[b] <orgDiv/>Nanobioelectronics and Biosensors Group, <orgName/>Catalan Institute of Nanoscience and Nanotechnology (ICN2), CSIC and The Barcelona Institute of Science and Technology

<street/>Campus UAB, <postCode/>08193<countryPart/>Bellaterra, <city/>Barcelona (<country/>Spain)

[c] <orgDiv/>School of Chemical Engineering $<$ orgName/>University of Adelaide < city/>Adelaide, <countryPart/>South Australia <postCode/>5005 (<country/>Australia) [d] <orgName/>ICREA <street/>Pg. Lluís Companys, 23, <city/>Barcelona <postCode/>08010 (<country/>Spain)

The prints and the paper: Print graphene on both sides of a common office paper acting as separator. Add an aqueous sulfuric acid electrolyte, current collectors and... Potassium Iodide as an electroactive redox couple for extra charge storage. The result is a low-cost microsupercapacitor with extra capacitance $\left(130^{\wedge \wedge} \mathrm{mF}^{\wedge} \mathrm{cm}^{<\mathrm{M}->3}\right)$ and energy density $\left(0.026^{\wedge \wedge} \mathrm{mWh}^{\wedge} \mathrm{cm}^{<\mathrm{M}->3}\right)$, ideally suited for flexible applications. 
Pedro Gomez-Romero et al. (@icn2nano) Design and Fabrication of Printed Paper-Based Hybrid Micro \#Supercapacitors by using Graphene and Redox-Active Electrolyte

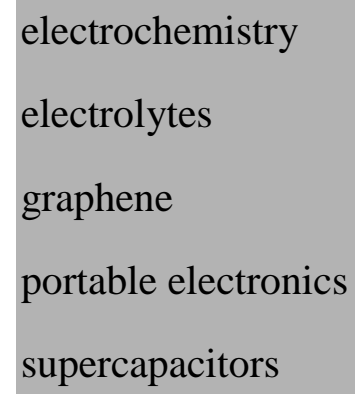

Inspired by future needs of flexible, simple, and low-cost energy storage devices, smart graphene-based micro-supercapacitors on conventional Xerox paper substrates were developed. The use of redox-active species (iodine redox couple) was explored to further improve the paper device's performance. The device based on printed graphene paper itself already had a remarkable maximum volumetric capacitance of $29.6^{\wedge \wedge} \mathrm{mF}^{\wedge} \mathrm{cm}^{<\mathrm{M}->3}$ (volume of whole device) at $6.5^{\wedge \wedge} \mathrm{mA}^{\wedge} \mathrm{cm}^{<\mathrm{M}->3}$. The performance of the hybrid electrode with redoxactive potassium iodide at the graphene surface was tested. Remarkably, the hybrid device showed improved volumetric capacitance of $130^{\wedge \wedge} \mathrm{mF}^{\wedge} \mathrm{cm}^{<\mathrm{M}->3}$. The maximum energy density for a graphene+KI device in $\mathrm{H}_{2} \mathrm{SO}_{4}$ electrolyte was estimated to be $0.026^{\wedge \wedge} \mathrm{mWh}^{\wedge} \mathrm{cm}^{<\mathrm{M}->3}$. Thus, this work offers a new simple, and lightweight micro-supercapacitor based on low-cost printed graphene paper, which will have great applications in portable electronics.

\section{Introduction}

The demand for highly efficient and cost-effective energy storage systems is on a steep rise. Consequently, research into energy storage technologies is trying to keep pace through the development of new materials and devices. Electrochemical energy storage devices----primarily batteries and supercapacitors----are some of the most promising technologies in this relentless race. The batteries work on a bulk storage mechanism, exhibiting a high energy density but a low power density and less cycling stability, whereas supercapacitors work on storing charges through non-faradaic surface adsorption and/or faradaic redox reactions (pseudocapacitive) and feature high power density with excellent cycling stabilities. ${ }^{[1, \wedge 2]}$ Graphene is a well-known two-dimensional $\left(2^{\wedge} \mathrm{D}\right)$ nanocarbon material 
with outstanding thermal and electrical conductivity and high mechanical and chemical stability. Owing to its high surface area, graphene has a very high inherent electric-doublelayer capacitance. ${ }^{[3]}$ The calculated theoretical specific capacitance of single-layered graphene is approximately $21^{\wedge \wedge} \mu \mathrm{F}^{\wedge} \mathrm{cm}^{<\mathrm{M}->2}$ or $550^{\wedge \wedge} \mathrm{F}^{\wedge} \mathrm{g}^{<\mathrm{M}->1} \cdot{ }^{[4]}$ In practice, however, these values are lower for several reasons, among which, the re-stacking of graphene layers, which hides and limits its active area, is one of the most detrimental. Thus, effective microstructuring of graphene electrodes is one of the primary approaches towards high-performance graphene supercapacitors. The other main approach is the reinforcement of its energy storage capability by hybridization, that is, through the incorporation of faradaic charge-storage components. Redox-active species can be incorporated into the device by means of three different approaches, namely, i $)^{\wedge \wedge}$ covalent attachment to graphene electrodes, ii $)^{\wedge \wedge}$ adsorption onto the capacitive carbon-based electrodes or iii)^^they can be added to the electrolyte, turning it into a hybrid redox-active electrolyte.

In this study, we designed a high-performance graphene micro-supercapacitor by tackling both the shaping of graphene electrodes by direct printing on a paper substrate and the improvement of energy storage by incorporation of a redox-active species, potassium iodide (KI). KI is a remarkably non-toxic and environmentally friendly redox-active species compared to other redox species used in energy storage research, like hydroquinone, catechol, ferro/ferricyanide etc. KI enhances the capacitance of the device by forming different redox pairs of iodide on the surface of the electrode $3^{\wedge} \mathrm{I}^{\langle\mathrm{M}->} / \mathrm{I}_{3}{ }^{<\mathrm{M}->}, 2^{\wedge} \mathrm{I}^{\langle\mathrm{M}->} / \mathrm{I}_{2}, 2^{\wedge} \mathrm{I}_{3}{ }^{\langle\mathrm{M}->} / 3^{\wedge} \mathrm{I}_{2}$ and $\mathrm{I}_{2} / 2^{\wedge} \mathrm{IO}_{3}^{<\mathrm{M}->} \cdot[5]$

Flexibility and portability are two widely expected characteristics for modern industrial electronic technologies, which enable the fabrication a variety of sophisticated applications, such as flexible touchscreens, implantable sensors, flexible mobile phones, and flexible OLEDs. ${ }^{\left[6,{ }^{\wedge}\right]}$ Paper is among the best-known substrate materials for such applications, as it is flexible, highly cost-effective, environmentally compatible, and easy to handle. It is composed of cellulose, with a size of $210^{\wedge \wedge} \mathrm{mm} \times 297^{\wedge \wedge} \mathrm{mm}$ (DinA4 paper) and diameter of $20^{\wedge} \wedge \mu \mathrm{m}$. Papers has found a wide range of applications in electronics, solar cells, and energy devices and is also highly suitable for printing. ${ }^{[8]}$ 
Various printing techniques have been used for the fabrication of flexible electrodes for energy storage application, including roll-to-roll, screen printing, inkjet printing, and transfer techniques. ${ }^{[9--11]}$ However, all of these printing methods have pros and cons and it is therefore very important to use the most appropriate printing technique for each specific application. In this study, a modified transfer technique using a wax printer and stamping mechanism was used in which a pattern was drawn over a nitrocellulose filter membrane and graphene was filtered through it and the pattern stamped over paper. This technique does not allow graphene sheets to move through the paper and they remain at the surface while the interior of the paper can be used as an electrolyte absorbent, as well as a separator. Thus, this strategy provides a unique means of fabrication for paper-based supercapacitors, which can be used in several portable and wearable applications.

We have designed and engineered a smart paper-based graphene supercapacitor device from the point of view of feasibility in industries that require easy and cheap fabrication along with enhanced supercapacitive properties in an aqueous electrolyte. The motivation is to enhance the capacitance and voltage of supercapacitors using aqueous electrolytes by shifting the oxygen evolution potential. In this context, we have used potassium iodide as a redoxactive species (iodide/iodine redox pair), which stimulates the capacitance and widen the voltage window. ${ }^{[12--15]}$ Most of the previous work has focused on the introduction of KI species in the electrolyte. ${ }^{[16--19]}$ However, in the present study, we have investigated the effect of KI addition on the surface of printed graphene electrodes.

\section{Results and Discussion}

\section{Morphological analysis}

The transmission electron microscopy (TEM) image of the graphene nanosheet (Figure ${ }^{\wedge \wedge} 1<$ figr $\left.1>\wedge a\right)$ shows that the nanosheets are thin and transparent with extra-large size and its selected-area electron-diffraction $(\mathrm{SAED})$ pattern (Figure ${ }^{\wedge} 1<x$ figr $\left.1>b\right)$ confirms the formation of graphene. Figure ${ }^{\wedge} 1^{\wedge} \mathrm{c}<\mathrm{xfigr} 1>$ shows the scanning electron microscopy (SEM) image of a usual copy paper printed with graphene from the top view and a cross sectional view. The morphology of the paper is very rough and we took the advantage of this surface 
roughness to build an energy storage device that can absorb more electrolyte than the semipermeable filter membranes and can hence provide increased access to the exposed stamp-printed graphene. The SEM image in Figure ${ }^{\wedge \wedge} 1^{\wedge} \mathrm{d}<\mathrm{xfigr} 1>$ shows the uniform printing of graphene sheets over the paper surface. With this printing technique, the graphene stays only at the surface of the paper and does not penetrate through to the other side, allowing us to stamp the prints on both sides of the paper, which serves as both a substrate and a separator (see cross-sectional view, Figure ${ }^{\wedge} 1^{\wedge} \mathrm{c}<\mathrm{xfigr} 1>$ ). Owing to strong binding forces in the paper, the adhesion of graphene layers to the paper is very strong and can be retained throughout a rubbing test.

Raman spectroscopy was used for verifying graphene layers. The distinctive $G$ and $2^{\wedge} \mathrm{D}$ peaks appeared at 1580 and $2702^{\wedge \wedge} \mathrm{cm}^{<\mathrm{M}->1}$, respectively, and correspond to few-layered graphene with a D-band at $1350^{\wedge \wedge} \mathrm{cm}^{<\mathrm{M}->1}$, indicating few defects (Figure ${ }^{\wedge \wedge} 1 \wedge \mathrm{e}<\mathrm{xfigr} 1>$ ). The $I_{\mathrm{G}} / I_{2 \mathrm{D}}$ ratio suggested the formation of few-layered graphene. ${ }^{[20]}$ The narrow $2 \mathrm{D}$ peak appears at $2700^{\wedge \wedge} \mathrm{cm}^{<\mathrm{M}->1}$, which is different from $2 \mathrm{D}$ peak of graphite as it is a mixture of $2 \mathrm{D}_{1}$ and $2 D_{2 \cdot}{ }^{[21, \wedge 22]}$ Moreover, the X-ray diffraction $(X R D)$ pattern (Figure $\left.{ }^{\wedge} 1^{\wedge} f<x f i g r 1>\right)$ showed a broad peak at $24.5^{\circ}$, which is associated with the characteristic (002) plane of graphene and represents an interlayer distance of $0.35^{\wedge \wedge} \mathrm{nm} .{ }^{[23]}$

Figure $^{\wedge \wedge} 2<$ figr $2>$ shows some of the steps involved in the printing and fabrication of a device. Figure ${ }^{\wedge \wedge} 2^{\wedge} \mathrm{a}--\mathrm{c}<x$ figr $2>$ shows photographs of graphene printed on both sides of the paper, suggesting a highly flexible electrode design to be used in supercapacitors.

Figure $\wedge^{\wedge} 2^{\wedge} \mathrm{d}<\mathrm{xfigr} 2>$ shows a schematic diagram of the supercapacitor cell assembly for testing.

\section{Electrochemical Testing}

All electrochemical testing of the device was performed in a two-electrode system by using $1^{\wedge} \mathrm{M} \mathrm{H}_{2} \mathrm{SO}_{4}$ electrolyte (see the Supporting Information, Figure ${ }^{\wedge} \mathrm{S} 1$ ). Figure ${ }^{\wedge} 3^{\wedge} \mathrm{a}<$ figr $3>$ shows the cyclic voltammetry $(\mathrm{CV})$ curves of the paper based microsupercapacitor device in a conventional $1^{\wedge} \mathrm{M} \mathrm{H}_{2} \mathrm{SO}_{4}$ electrolyte at various scan rates ranging from 5 to $100^{\wedge \wedge} \mathrm{mV}^{\wedge} \mathrm{s}^{<\mathrm{M}->1}$ in the voltage range of $0--0.8^{\wedge} \mathrm{V}$. The $\mathrm{CV}$ curve shapes are ideally rectangular with perfect symmetry, which corresponds to the inherent electric-double-layer 
capacitance (EDLC) of graphene sheets. The paper substrate did not interfere in the performance of the device and the capacitance increased with increasing scan rate. To assess the overall device performance in terms of energy storage, galvanostatic charge--discharge measurements were carried out at current densities from 6.5 to $10.5^{\wedge \wedge} \mathrm{mA}^{\wedge} \mathrm{cm}^{<\mathrm{M}->3}$ (Figure ${ }^{\wedge \wedge} 3^{\wedge} b<x f i g r 3>$ ). The volumetric capacitance calculated from charge--discharge curves [see Experimental Section, Eqs. ${ }^{\wedge \wedge}(5)$ and (6)] were $15.48,19.6,24$, and $29.6^{\wedge \wedge} \mathrm{mF}^{\wedge} \mathrm{cm}^{<\mathrm{M}->3}$ at current densities of $6.5,7.8,9.2$, and $10.5^{\wedge \wedge} \mathrm{mA}^{\wedge} \mathrm{cm}^{<\mathrm{M}->3}$, respectively (areal capacitances were $1.2,1.4,1.8$, and $2.2^{\wedge \wedge} \mathrm{mF}^{\wedge} \mathrm{cm}^{<\mathrm{M}->2}$ at $0.79,0.69,0.59$, and $0.49^{\wedge \wedge} \mathrm{mA}^{\wedge} \mathrm{cm}^{<\mathrm{M}->2}$, respectively). There is a decrease in the capacitance with applied current density with a maximum volumetric capacitance of $29.6^{\wedge \wedge} \mathrm{mF}^{\wedge} \mathrm{cm}^{<\mathrm{M}->3}$ (areal capacitance was $2.2^{\wedge \wedge} \mathrm{mF}^{\wedge} \mathrm{cm}^{<\mathrm{M}->2}$ ) at $6.5^{\wedge \wedge} \mathrm{mA}^{\wedge} \mathrm{cm}^{<\mathrm{M}->3}$ in $\mathrm{H}_{2} \mathrm{SO}_{4}$ electrolyte. The calculated capacitance is significantly higher than that for some other reported supercapacitor devices. For example, $\mathrm{Wu}$ et ${ }^{\wedge \wedge}$ al. reported a capacitance of $0.95^{\wedge \wedge} \mathrm{mF}^{\wedge} \mathrm{cm}^{<\mathrm{M}->2}$ for an interdigitated reduced graphene oxide hydrogel-patterned micro-supercapacitor over a metallic substrate. ${ }^{[24]}$ Pech and co-workers reported a capacitance of $2.1^{\wedge \wedge} \mathrm{mF}^{\wedge} \mathrm{cm}^{<\mathrm{M}->2}$ for an inkjet-printed graphene micro-supercapacitor with an $\mathrm{Et}_{4} \mathrm{NBF}_{4}$ electrolyte, ${ }^{[25]}$ whereas Bae et ${ }^{\wedge \wedge}$ al. attained around $0.4^{\wedge \wedge} \mathrm{mF}^{\wedge} \mathrm{cm}^{<\mathrm{M}->2}$ areal capacitance by using graphene and $\mathrm{ZnO}$ nanowires. ${ }^{[26]}$ Similarly, Gao et ${ }^{\wedge}$ al. developed a flexible solid-state graphene supercapacitor with a capacitance of $12.4^{\wedge \wedge} \mu \mathrm{F}^{\wedge} \mathrm{cm}^{<\mathrm{M}->2} \cdot{ }^{[27]}$

The general goal of hybrid materials design is to combine the properties and identify synergies between two different materials. In energy storage devices, hybrid materials enhance the electrochemical performance of the device by complementing capacitive with pseudocapacitive materials. ${ }^{[2]}$ In the present work, we adsorbed a redox-active species---particular potassium iodide----on the surface of graphene to improve the performance of the final device. We tested three different concentrations of KI adsorbed onto graphene and compared the results to those for the graphene $+\mathrm{H}_{2} \mathrm{SO}_{4}$ system. To confirm the presence and adsorption retention of iodide species on the surface of the graphene electrode, we carried out X-ray photoelectron spectroscopy (XPS) of our positive (working) electrode at different stages, that is, before cycling, after the first charge, and after the first discharge (Figure ${ }^{\wedge \wedge} 4^{\wedge} \mathrm{a}, \wedge \mathrm{b}<$ figr $4>$ ). The XPS spectra suggest the presence of $\mathrm{C}, \mathrm{O}$, and I peaks in all 
three graphene samples (Figure ${ }^{\wedge \wedge} 4^{\wedge} \mathrm{a}<x$ figr $4>$ ). The two apparent $\mathrm{I}^{\wedge} 3 \mathrm{~d}$ peaks at 619.4 and $631.0^{\wedge} \mathrm{eV}$ can be assigned to $\mathrm{I}^{\wedge} 3 \mathrm{~d}_{5 / 2}$ and $\mathrm{I}^{\wedge} 3 \mathrm{~d}_{3 / 2}$ states, respectively (Figure $\wedge^{\wedge} 4^{\wedge} \mathrm{b}<x$ figr $4>$ ). The signal for $\mathrm{I}^{\wedge} 3 \mathrm{~d}_{5 / 2}$ at $619.6^{\wedge \wedge} \mathrm{eV}$ correspond to bonding between carbon and iodine species, such as $\mathrm{C}<\mathrm{C}->\mathrm{I}$ and $\mathrm{C}<\mathrm{C}->\mathrm{I}^{+}<\mathrm{C}->\mathrm{C}$ as the oxidation products of $\mathrm{I}^{<\mathrm{M}->}$ during the reaction process. ${ }^{\left[28,{ }^{\wedge 29}\right]}$ The amounts of iodide species adsorbed onto the graphene surface were found to be $86^{\wedge \wedge}$ at ${ }^{\wedge} \%$, which slightly decreased after the first charge (to around $78^{\wedge \wedge}$ at ${ }^{\wedge} \%$ ) and thereafter remained constant (at around $77^{\wedge \wedge} \mathrm{at} \mathrm{t}^{\wedge} \%$ ). These results suggest that only a very small percentage of the iodide species dissolved in the acidic electrolyte in the beginning, and the amount remained constant thereafter, indicating a very efficient attachment of iodide on the graphene surface. The electrochemical performances of the as-assembled device with iodide species adsorbed on graphene are presented in Figure ${ }^{\wedge} 5<$ figr5> (for further details, see Figures $\left.{ }^{\wedge} \mathrm{S} 2--\mathrm{S} 4\right)$. Figure ${ }^{\wedge \wedge} 5^{\wedge} \mathrm{a}<\mathrm{x}$ figr5 $>$ shows the cyclic voltammetry (CV) curves for printed graphene device in conventional $\mathrm{H}_{2} \mathrm{SO}_{4}$ and $\mathrm{KI}$-doped graphene electrode at a scan rate of $10^{\wedge \wedge} \mathrm{mV}^{\wedge} \mathrm{s}^{<\mathrm{M}->1}$. The area under the $\mathrm{CV}$ curves increases significantly with the concentration of KI, suggesting a remarkable increase in the capacitance of the device. The device can be easily cycled up to $1.2^{\wedge \wedge} \mathrm{V}$, whereas for conventional electrochemical capacitors with aqueous electrolytes the working voltage range varies from 0.6 to $0.8^{\wedge \wedge} \mathrm{V} \cdot{ }^{[30]}$ Moreover, the shapes of the CV curves are distorted from the ideal rectangular shapes typical of electric-double-layer capacitors (carbon materials), which confirms the involvement of redox-active species (iodine/iodide) at the electrode/electrolyte interface.

The charge storage mechanism of paper-based hybrid micro-supercapacitors is explained as follows: After initiation of the reaction (providing the required potential to the iodide species), both reactants and products $\left(\mathrm{I}^{<\mathrm{M}->}\right.$ and $\mathrm{I}_{3}^{<\mathrm{M}->}$ ions $)$ are negatively charged in the aqueous electrochemical oxidation of iodides. These species balance the charges in the electrochemical double layer (EDL) while electrostatically remaining in the positively charged electrode, hence increasing the charge storage capacity. The CV curves show the iodide redox reaction peaks, which are the source of the extra (redox) capacity and can be assigned to the reactions given by Equations ${ }^{\wedge \wedge}(1<\mathrm{ffr} 1>)--(4<\mathrm{ffr} 2><\mathrm{ffr} 3><\mathrm{ffr} 4>)$ (with Eq. ${ }^{\wedge}(2)$ predominating in aqueous media): ${ }^{[12]}$ 


$$
\begin{aligned}
& <\mathrm{ff} 1>2^{\wedge} \mathrm{I}^{<\mathrm{M}->} \rightarrow \mathrm{I}_{2}+2^{\wedge} \mathrm{e}^{\langle\mathrm{M}->}<\mathrm{ZS}>(1) \\
& \left\langle\mathrm{ff} 2>3^{\wedge} \mathrm{I}^{<\mathrm{M}->} \rightarrow \mathrm{I}_{3}^{<\mathrm{M}->}+2^{\wedge} \mathrm{e}^{\langle\mathrm{M}->}<\mathrm{ZS}>(2)\right. \\
& <\mathrm{ff} 3>2^{\wedge} \mathrm{I}_{3}{ }^{<\mathrm{M}->} \rightarrow 3 \mathrm{I}_{2}+2^{\wedge} \mathrm{e}^{\langle\mathrm{M}->}<\mathrm{ZS}>(3) \\
& <\mathrm{ff} 4>\mathrm{I}_{2}+6^{\wedge} \mathrm{H}_{2} \mathrm{O} \rightarrow 2^{\wedge} \mathrm{IO}_{3}{ }^{<\mathrm{M}->}+12^{\wedge} \mathrm{H}^{+}+10^{\wedge} \mathrm{e}^{<\mathrm{M}->}<\mathrm{ZS}>(4)
\end{aligned}
$$

Considering that the ions can turn into polyions, the possibility of formation of polyiodides $\left(\mathrm{I}_{3}{ }^{<\mathrm{M}->} / \mathrm{I}_{5}{ }^{<\mathrm{M}->}\right)$ cannot be ruled out, but, according to the Pourbaix diagram for iodine ${ }^{\left[31,{ }^{\wedge 2}\right]}$ the possibility of their formation is low (it is more favorable in alkaline medium and at slightly higher potentials) and also the iodides have limited tendency for solvation. Moreover, the increase in the operating voltage can be further ascribed to the presence of KI, as reported by Fic et ${ }^{\wedge \wedge}$ al. ${ }^{[33]}$ The addition of KI extends the hydrogen evolution potential of the working electrode in the negative region and shows redox activity in the positive range. In the present investigation, we took advantage of this characteristic of KI and obtained an extended voltage window for our printed graphene device, although, with the extension of potential at the positive end, the hydrogen storage process becomes aggravated and the total potential window is compromised. However, these ranges can be further enhanced by using asymmetric electrodes, different electrode materials or electrolytes. ${ }^{[34--36]}$

The galvanostatic charge--discharge curves recorded at $6.5^{\wedge \wedge} \mathrm{mA}^{\wedge} \mathrm{cm}^{<\mathrm{M}->3}$ (Figure $\wedge^{\wedge} 5^{\wedge} \mathrm{b}<\mathrm{xfigr} 5>$ ) are in good agreement with the CV results (Figure ${ }^{\wedge \wedge} 5^{\wedge} \mathrm{a}<\mathrm{xfigr5}>$ ). The device with $2^{\wedge} \mathrm{M} \mathrm{KI}$ showed a prolonged discharge time over those with other concentrations, indicating high energy and capacitance. The maximum volumetric capacitance obtained for graphene device with $2^{\wedge} \mathrm{M} \mathrm{KI}$ is $130^{\wedge \wedge} \mathrm{mF}^{\wedge} \mathrm{cm}^{<\mathrm{M}->3}\left(9.8^{\wedge \wedge} \mathrm{mF}^{\wedge} \mathrm{cm}^{<\mathrm{M}->2}\right)$, which is almost $5^{\wedge \wedge}$ times higher than that for the conventional graphene electrode (Figure $\wedge^{\wedge} 5^{\wedge} \mathrm{c}<\mathrm{xfigr} 5>$ ). In addition, the device achieved maximum energy and power density values of $0.026^{\wedge \wedge} \mathrm{mWh}^{\wedge} \mathrm{cm}^{<\mathrm{M}->3}$ and $13.38^{\wedge \wedge} \mathrm{mW}^{\wedge} \mathrm{cm}^{<\mathrm{M}->3}$, respectively, in the presence of $2^{\wedge} \mathrm{M} \mathrm{KI}+$ graphene in $\mathrm{H}_{2} \mathrm{SO}_{4}$ (Figure ${ }^{\wedge} 5^{\wedge} \mathrm{d}<\mathrm{xfigr5}>$ ). Among the three concentrations, the device showed maximum efficiency with $2^{\wedge} \mathrm{M} \mathrm{KI}$ added to $\mathrm{H}_{2} \mathrm{SO}_{4}$. To our knowledge, the maximum capacitance and the working potential range observed here are the highest reported to date for a paper-based graphene micro-supercapacitor device with aqueous electrolyte. 
Electrochemical impedance spectroscopy (EIS) was carried out on the devices in the frequency range of $100^{\wedge \wedge} \mathrm{kHz}--100^{\wedge \wedge} \mathrm{mHz}$ at electrical open-circuit potential. The Nyquist plot obtained for the micro-supercapacitor device (Figure ${ }^{\wedge \wedge} 6^{\wedge} \mathrm{a}\langle$ figr6 $>$ ) indicates that the devices acts like a pure capacitor without any sign of resistor-like behavior that blocks the transfer of charges at the electrode/electrolyte interface. In theory, a vertical line at $90^{\circ}$ must be observed as a sign of pure capacitor, but somehow due to few properties of the electrode like roughness, porosity etc., there can be some deviation from the vertical line. Roughness arising from the paper, addition of redox-active species and the pore size of graphene could be other possible reasons for non-vertical low frequency signal in this case. The Bode plot (Figure ${ }^{\wedge \wedge} 6^{\wedge} b<x$ figr6>) provides information of the phase-angle dependence on frequency. The phase angle approaches $73^{\circ}$ in the case of graphene, for which EDLC is the sole mechanism of charge storage, whereas after addition of redox species the phase angles shift to $61^{\circ}, 67^{\circ}$, and $65^{\circ}$ for $0.5^{\wedge} \mathrm{M} \mathrm{KI}, 1^{\wedge} \mathrm{M} \mathrm{KI}$, and $2^{\wedge} \mathrm{M} \mathrm{KI}$, respectively, indicating the dominance of faradaic processes as the charge storage mechanism. The phase angle approaching $90^{\circ}$ represents ideal capacitive behavior and the frequency at which the phase angle crosses $45^{\circ}$ is associated to the capacitive behavior of the graphene electrodes. The graphene electrode crosses $45^{\circ}$ at a frequency of $1.1^{\wedge \wedge} \mathrm{Hz}$, which already shows a very fast frequency response, whereas the graphene with iodide species crosses $45^{\circ}$ at even lower frequencies of 1.05, 1.05, and 0.85 for $0.5^{\wedge} \mathrm{M}, 1^{\wedge} \mathrm{M}$, and $2^{\wedge} \mathrm{M} \mathrm{KI}$, respectively, suggesting a swift frequency response owing to adsorbed iodide and excellent capacitive behavior. ${ }^{[37]}$ Additionally, EIS can be used to study the electrochemical behavior of the fabricated device by using Equations ${ }^{\wedge \wedge}(5<\mathrm{ffr} 5>)$ $-(7<\mathrm{ffr} 6><\mathrm{ffr} 7>)$ to calculate the real and imaginary capacitances at the corresponding frequencies:

$$
\begin{aligned}
& <\mathrm{ff} 5>C(\omega)=C^{\prime}(\omega)<\mathrm{M}->j C^{\prime \prime}(\omega)<\mathrm{ZS}>(5) \\
& <\mathrm{ff} 6>C^{\prime}(\omega)=\frac{Z^{\prime \prime}(\omega)}{\omega|Z(\omega)|^{2}}<\mathrm{ZS}>(6) \\
& <\mathrm{ff} 7>C^{\prime \prime}(\omega)=\frac{Z^{\prime}(\omega)}{\omega|Z(\omega)|^{2}}<\mathrm{ZS}>(7)
\end{aligned}
$$

where $Z$ is the complex impedance given by $Z(\omega)=Z^{\prime}(\omega)+Z^{\prime \prime}(\omega)$ and $\omega=2 \pi f, f$ is the frequency, $Z^{\prime}$ and $Z^{\prime}$ are the real and imaginary parts of the Nyquist plot and $C^{\prime \prime}$ and $C^{\prime \prime}$ are the real and 
imaginary capacitances. In the plot of capacitance vs. frequency (Figure $\wedge^{\wedge} 6^{\wedge} c<x f i g r 6>$ ), the capacitance decreases with increasing frequency. We determined the relaxation time constant $\left(\tau_{\mathrm{e}}\right)$ by using Equation ${ }^{\wedge}(8<\mathrm{ffr} 8>)$ :

$<\mathrm{ff} 8>\tau_{\mathrm{e}}=\frac{1}{f_{\mathrm{e}}}<\mathrm{ZS}>(8)$

where $\tau_{\mathrm{e}}$ is the minimum time needed by the device to discharge all of its energy with more than $50^{\wedge} \%$ efficiency of the maximum value and $f_{\mathrm{e}}$ is the frequency. It is taken from the frequency at maximum $C^{\prime \prime} .^{\left[38,{ }^{\wedge 39]}\right.}$ The lowest relaxation time constant was calculated for graphene electrodes to be $200^{\wedge \wedge} \mathrm{ms}$. This value is significantly lower than those for supercapacitors based on activated carbon $\left(700^{\wedge \wedge} \mathrm{ms}, 4.3^{\wedge \wedge} \mathrm{s}\right),{ }^{[40]}$ reduced graphene oxide $\left(1.034^{\wedge \wedge} \mathrm{s}, 430^{\wedge \wedge} \mathrm{ms}\right),{ }^{[41--43]}$ and hybrid graphene $\left(392^{\wedge \wedge} \mathrm{ms}\right) \cdot{ }^{[44]}$ The long-term cycling stability was investigated at a current density of $2^{\wedge \wedge} \mathrm{mA}$ for 3000 galvanostatic charge-discharge cycles (Figure ${ }^{\wedge \wedge} 7<$ figr $7>$ ). Around $97^{\wedge} \%$ capacitance retention over 3000 cycles for the device with graphene in $\mathrm{H}_{2} \mathrm{SO}_{4}$ electrolyte was observed. In contrast, the capacitance declines quickly for graphene electrodes with KI added graphene SCs $\left(90.4^{\wedge} \%, 84^{\wedge} \%\right.$, and $65^{\wedge} \%$ capacitance retention with $0.5^{\wedge} \mathrm{M}, 1^{\wedge} \mathrm{M}$, and $2^{\wedge} \mathrm{M} \mathrm{KI}$, respectively). This decrease in the capacitance might be attributed to the redox transitions of iodide species during the charge-discharge process.

These results demonstrate the feasibility of using a simple technique with costeffective materials to fabricate highly efficient supercapacitors. In addition to the excellent electrochemical performances, this work opens a pool of possibilities to aiding advancements in the field of flexible paper-based devices. For example, the performances can be further improved by using different redox-active electrolytes, graphene based hybrids composites electrodes, different quality of papers and fabrication of handy pouch devices.

\section{Conclusions}

We have designed and engineered a paper-based graphene micro-supercapacitor device by introducing the redox-active species KI onto the surface of graphene electrodes. This strategy not only enhanced the capacitance but also extended the working voltage range, eventually leading to improved energy and power densities. The paper simultaneously served 
three functions: as substrate, separator, and electrolyte absorbent without any pre-treatment. This device is fabricated by using a facile stamping technique and provided a maximum volumetric capacitance of $130^{\wedge \wedge} \mathrm{mF}^{\wedge} \mathrm{cm}^{<\mathrm{M}->3}$ (areal capacitance of $10^{\wedge \wedge} \mathrm{mF}^{\wedge} \mathrm{cm}^{<\mathrm{M}->2}$ ) at $6.5^{\wedge \wedge} \mathrm{mA}^{\wedge} \mathrm{cm}^{<\mathrm{M}->3}$. Moreover, the maximum energy and power densities were calculated to be $0.026^{\wedge \wedge} \mathrm{mWh}^{\wedge} \mathrm{cm}^{<\mathrm{M}->3}$ and $13.4^{\wedge \wedge} \mathrm{mW}^{\wedge} \mathrm{cm}^{<\mathrm{M}->3}$, respectively. The device exhibited very good cycling stability of around $97^{\wedge} \%$ capacitance retention over 3000 cycles. Since research on paper-based supercapacitor devices is in its early stages compared to that on devices based on other flexible substrates, these results show a very good combination of EDLC and redox mechanism by using this hybrid electrode concept, which opens a door for further research in this area.

\section{Methods}

\section{Materials and reagents}

Pristine graphene powder $\left(<20^{\wedge \wedge} \mu \mathrm{m}\right)$, potassium iodide $\left(\geq 99.0^{\wedge} \%\right)$, and sulfuric acid (99.999^\%) were purchased from Sigma Aldrich. Nitrocellulose filter membranes (pore size $=0.025^{\wedge} \mu \mathrm{m}$ ) were purchased from MF-Milipore, Merck.

\section{Fabrication of graphene--KI hybrid}

Graphene was synthesized by using similar procedure to that described in Ref. $\wedge^{\wedge}<$ litrsn $><\operatorname{litr} 45></$ litrsn $>$. A $0.25^{\wedge \wedge} \mathrm{mg}^{\wedge} \mathrm{mL}^{<\mathrm{M}->1}$ graphene dispersion was prepared by sonication. $0.5^{\wedge} \mathrm{M}, 1^{\wedge} \mathrm{M}$, or $2^{\wedge} \mathrm{M}$ KI was then added to the graphene suspension and the mixture sonicated for $1^{\wedge \wedge} \mathrm{h}$ and left to settle overnight. The sediment was filtered off and re-dispersed in deionized water.

$<+>$ Stamp printing was used for printing of graphene on both sides of the paper by the method described in Ref. $\wedge^{\wedge}<$ litrsn $><$ litr46></litrsn $>$. Briefly, graphene was dispersed in water at a concentration of $0.25^{\wedge \wedge} \mathrm{mg}^{\wedge} \mathrm{mL}^{<\mathrm{M}->1}$ and filtered through a hydrophilic $0.025^{\wedge \wedge} \mu \mathrm{m}$ pore size nitrocellulose filter membrane with a diameter of $47 \mathrm{~mm}$ having had a pattern printed on it by using a Xerox ColorQube 8570 printer. This pattern was then transferred onto the paper by a roll-to-roll mechanism (built into the printer) 


\section{Fabrication and performance evaluation of graphene device}

The printed electrodes were soaked in either conventional $\mathrm{H}_{2} \mathrm{SO}_{4}$ or $\mathrm{KI}$-added $\mathrm{H}_{2} \mathrm{SO}_{4}$ for $5^{\wedge \wedge} \min$ before each measurement. Carbon cloth was used as the current collector and was placed on both sides of the paper. The device was covered with a nonconducting parafilm tape and a clip was used to hold the device together before making connections for electrochemical measurements.

$<+>$ A Biologic potentiostat/galvanostat was used for all electrochemical measurements within the voltage range of $0--1.2^{\wedge \wedge} \mathrm{V}$. Electrochemical impedance spectroscopy (EIS) was carried in the frequency range of $100^{\wedge} \mathrm{kHz}--100^{\wedge \wedge} \mathrm{mHz}$. The areal capacitance $\left(C_{\mathrm{a}}\right)$ and volumetric capacitance $\left(C_{\mathrm{v}}\right)$ of a two-electrode cell was calculated by using Equations ${ }^{\wedge \wedge}(9<\mathrm{ffr} 9>)$ and $(10<\mathrm{ffr} 10>)$ :

$<\mathrm{ff} 9>C_{\mathrm{V}}=\frac{I \times A_{\mathrm{cd}}}{V_{\mathrm{d}} \times V^{2}}<\mathrm{ZS}>(9)$

$<\mathrm{ff} 10>C_{\mathrm{a}}=\frac{I \times A_{\mathrm{cd}}}{A_{\mathrm{d}} \times V^{2}}<\mathrm{ZS}>(10)$

where $I$ is the current, $t$ is the time, $A_{\mathrm{cd}}$ is the area of the charge--discharge curve, $V_{\mathrm{d}}$ and $A_{\mathrm{d}}$ are the volume and area of device, respectively, $V$ represents the working voltage window. The areal and volumetric energy densities $\left(E_{\mathrm{a}}\right.$ and $\left.E_{\mathrm{v}}\right)$ and areal and volumetric power densities $\left(P_{\mathrm{a}}\right.$ and $\left.P_{\mathrm{v}}\right)$ were calculated by using Equations ${ }^{\wedge \wedge}(11<\mathrm{ffr} 11>)$ and $\left.(12<\mathrm{ffr} 12\rangle\right)$ :

$<\mathrm{ff} 11>E_{\mathrm{a}}=\frac{1}{2} C_{\mathrm{a}} \times V^{2}, \ldots \quad E_{\mathrm{v}}=\frac{1}{2} C_{\mathrm{v}} \times V^{2}\langle\mathrm{ZS}\rangle(11)$

$<\mathrm{ff} 12>P_{\mathrm{a}}=\frac{E_{\mathrm{a}}}{t}, \quad P_{\mathrm{v}}=\frac{E_{\mathrm{V}}}{t}<\mathrm{ZS}>(12)$

\section{Acknowledgements}

Partial funding from MINECO (grants no. MAT2015-68394-R, and MAT2017-87202-P MINECO/FEDER) and recognition from AGAUR (2017_SGR_870) are acknowledged. The ICN2 is supported by the Severo Ochoa program of the Spanish Ministry of Economy, Industry, and Competitiveness (MINECO, grant no. SEV-2013-0295) and funded by the CERCA program/ Generalitat de Catalunya 
$<$ lit1><lit_a $><$ jnl $>$ P.^^H. Yang, W.^^J. Mai, Nano Energy 2014, 8, 274--290</jnl>;

$<$ lit_b $><$ jnl $>$ D. ${ }^{\wedge}$ P. Dubal, N. ${ }^{\wedge}$ R. Chodankar, D. ${ }^{\wedge}$ H. Kim, P. Gomez-Romero, Chem. Soc. Rev. 2018, 47, 2065--2129</jnl>.

$<$ lit2><jnl>D.^^P. Dubal, O. Ayyad, V. Ruiz, P. Gomez-Romero, Chem. Soc. Rev. 2015, 44, $1777--1790</$ jnl $>$.

$<$ lit3><jnl>Q. Ke, J. Wang, J. Materiomics 2016, 2, 37--54</jnl >.

$<$ lit4><jnl>J. Xia, F. Chen, J. Li, N. Tao, Nat. Nanotechnol. 2009, 4, 505--509</jnl>.

$<$ lit5><jnl >D. Madhabi, A. Kumar, J. Phys. D 2018, 51, 085501</jnl >.

$<$ lit6><jnl >Y. Xu, Z. Lin, X. Huang, Y. Wang, Y. Huang, X. Duan, Adv. Mater. 2013, 25, $5779--5784</$ jnl $>$.

$<$ lit7><jnl $>$ W. ${ }^{\wedge}$ K. Chee, H.^^N. Lim, Z. Zainal, N. ${ }^{\wedge}$ M. Huang, I. Harrison, Y. Andou, J. Phys. Chem. C 2016, 120, 4153--4172</jnl>.

$<$ lit8 $>\left\langle\right.$ jnl $>Y .^{\wedge}$ Z. Zhang, Y. Wang, T. Cheng, W.^^Y. Lai, H. Pang, W. Huang, Chem. Soc. Rev. 2015, 44, 5181--5199</jnl>.

$<$ lit9><jnl >S. Lawes, A. Riese, Q. Sun, N. Cheng, X. Sun, Carbon 2015, 92, 150--176</jnl>. $<$ lit10><book>B. Roth, R.^^R. Søndergaard, F.^^C. Krebs in Handbook of Flexible Organic Electronics (Ed.: S. Logothetidis), Woodhead, Oxford, 2015, pp.^^171--197</book>. $<$ lit11><jnl>E.^^B. Secor, P.^^L. Prabhumirashi, K. Puntambekar, M.^^L. Geier, M.^^C. Hersam, J. Phys. Chem. Lett. 2013, 4, 1347--1351</jnl>.

$<$ lit12 $><$ jnl $>$ F. Béguin, V. Presser, A. Balducci, E. Frackowiak, Adv. Mater. 2014, 26, 2219-$2251</$ jnl $>$.

$<$ lit13><jnl>E. Frackowiak, M. Meller, J. Menzel, D. Gastol, K. Fic, Faraday Discuss. 2014, $172,179--198</$ jnl $>$.

<lit14><jnl>S. Roldán, Z. Gonzalez, C. Blanco, M. Granda, R. Menendez, R. Santamaria, Electrochim. Acta 2011, 56, 3401--3405</jnl>.

$<$ lit15 $><$ jnl $>$ A. Singh, A. Chandra, Sci. Rep. 2016, 6, 25793</jnl $>$. 
$<$ lit16><jnl >X. Cai, X. Cui, L. Zu, Y. Zhang, X. Gao, H. Lian, Y. Liu, X. Wang, Polymers 2017, $9,288</$ jnl $>$.

$<$ lit17 $><$ jnl $>$ D. Reber, R.-S. Kühnel, C. Battaglia, Sustainable Energy Fuels 2017, 1, 2155-$2161</$ jnl $>$.

$<$ lit18><jnl>D. Xu, W. Hu, X.^^N. Sun, P. Cui, X.^^Y. Chen, J. Power Sources 2017, 341, $448--456</$ jnl $>$.

$<$ lit19><jnl>Y. Zhang, L. Zu, H. Lian, Z. Hu, Y. Jiang, Y. Liu, X. Wang, X. Cui, J. Alloys Compd 2017, 694, 136--144</jnl>.

$<$ lit20><jnl>W. Wang, S. Guo, M. Penchev, I. Ruiz, K.^^N. Bozhilov, D. Yan, M. Ozkan, C.^^S. Ozkan, Nano Energy 2013, 2, 294--303</jnl>.

$<$ lit21 ><jnl>A.^^C. Ferrari, Solid State Commun. 2007, 143, 47--57</jnl>.

$<$ lit22><jnl>L.^^M. Malard, M.^^A. Pimenta, G. Dresselhaus, M.^^S. Dresselhaus, Phys. Rep. 2009, 473, 51--87</jnl>.

$<$ lit23><jnl> T. Lan, H. Qiu, F. Xie, J. Yang, M. Wei, Sci. Rep. 2015, 5, 8498</jnl>. $<$ lit24><jnl>Z.-K. Wu, Z. Lin, L. Li, B. Song, K.-S. Moon, S.-L. Bai, C.-P. Wong, Nano Energy 2014, 10, 222--228</jnl >.

$<$ lit25><jnl>D. Pech, M. Brunet, P.-L. Taberna, P. Simon, N. Fabre, F. Mesnilgrente, V.

Conedera, H. Durou, J. Power Sources 2010, 195, 1266--1269</jnl>.

$<$ lit26><jnl>J. Bae, Y.^^J. Park, M. Lee, S.^^N. Cha, Y.^^J. Choi, C. ${ }^{\wedge}$ S. Lee, J. ${ }^{\wedge}$ M. Kim, Z.^^L. Wang, Adv. Mater. 2011, 23, 3446--3449</jnl>.

$<$ lit27><jnl>Y. Gao, Y.^^S. Zhou, W. Xiong, L.^^J. Jiang, M. Mahjouri-samani, P.

Thirugnanam, X. Huang, M.^^M. Wang, L. Jiang, Y.^^F. Lu, APL Mater. 2013, 1, $012101</$ jnl $>$.

$<$ lit28 $><$ jnl $>$ L. Demarconnay, E. Raymundo-Piñero, F. Béguin, Electrochem. Commun. 2010, $12,1275--1278</$ jnl $>$. 
$<$ lit29><jnl>K. Jayaramulu, D.^^P. Dubal, B. Nagar, V. Ranc, O. Tomanec, M. Petr,

K. ${ }^{\wedge}$ K. ${ }^{\wedge}$ R. Datta, R. Zboril, P. Gómez-Romero, R.^^A. Fischer, Adv. Mater. 2018, 30, $1705789</$ jnl $>$.

$<$ lit30><jnl>C. Zhong, Y. Deng, W. Hu, J. Qiao, L. Zhang, J. Zhang, Chem. Soc. Rev. 2015, 44, 7484--7539</jnl >

$<$ lit31><other $>$ Y. Liu, H. ${ }^{\wedge}$ R. von ${ }^{\wedge}$ Gunten, Migration Chemistry and Behaviour of Iodine Relevant to Geological Disposal of Radioactive Wastes: A Literature Review with a Compilation of Sorption Data, PSI Bericht No. 16, 1988,

<url>https://www.nagra.ch/data/documents/database/dokumente/\$default/Default\%20F older/Publikationen/NTBs\%201987-1988/e<?_>ntb88-29.pdf</url $></$ other $>$.

<lit32><book> F.P Glasser in Chemistry and Microstructure of Solidified Waste Forms (Ed.:

R.^^D. Spence), CRC Press, Boca Raton, FL, 1993, Chapter 1, pp. 1-40</book>. $<$ lit33><jnl>K. Fic, M. Meller, E. Frackowiak, J. Electrochem. Soc. 2015, 162, A5140-A5147</jnl $>$.

$\left\langle\right.$ lit34 $>\left\langle\right.$ jnl $>$ N. ${ }^{\wedge}$ R. Chodankar, D. ${ }^{\wedge}$ P. Dubal, A. ${ }^{\wedge}$ C. Lokhande, A. ${ }^{\wedge \wedge}$ M. Patil, J. ${ }^{\wedge}$ H. Kim, C.^^D. Lokhande, Sci. Rep. 2016, 6, 39205</jnl>.

$<$ lit35><jnl>J. Menzel, K. Fic, E. Frackowiak, Prog. Nat. Sci. 2015, 25, 642--649</jnl>. $<$ lit36><jnl>E. Frackowiak, K. Fic, M. Meller, G. Lota, ChemSusChem 2012, 5, 1181-$1185</$ jnl $>$.

<lit37><jnl>D.^^P. Dubal, N.^^R. Chodankar, R. Holze, D.-H, Kim, P. Gomez-Romero, ChemSusChem 2017, 10, 1771--1782</jnl>.

$<$ lit38><jnl >K. Byungwoo, C. Haegeun, K. Woong, Nanotechnology 2012, 23, 155401</jnl>. $<$ lit39><jnl>K. Sheng, Y. Sun, C. Li, W. Yuan, G. Shi, Sci. Rep. 2012, 2, 247</jnl>. $<$ lit40><jnl>T.^^M. Masikhwa, M.^^J. Madito, D.^^Y. Momodu, J.^^K. Dangbegnon, O. Guellati, A. Harat, M. Guerioune, F. Barzegar, N. Manyala, RSC Adv. 2016, 6, 46723-$46732</$ jnl $>$. 
$<$ lit41 ><jnl>K. Lee, D. Kim, Y. Yoon, J. Yang, H.-G. Yun, I.-K. You, H. Lee, $R S C A d v$. 2015, 5, 60914--60919</jnl>.

$<$ lit42><jnl >A. Muthurasu, P. Dhandapani, V. Ganesh, New J. Chem. 2016, 40, 9111-$9124</$ jnl $>$.

$<$ lit43><jnl>K.^^P. Singh, D. Bhattacharjya, F. Razmjooei, Y.-S. Yu, Sci. Rep. 2016, 6, $31555</$ jnl $>$.

$<$ lit44><jnl>J. Yan, W. Sun, T. Wei, Q. Zhang, Z. Fan, F. Wei, J. Mater. Chem. 2012, 22, 11494--11502</jnl $>$.

$<$ lit45 $><$ jnl $>$ H. Zhu, Y. Cao, J. Zhang, W. Zhang, Y. Xu, J. Guo, W. Yang, J. Liu, J. Mater. Sci. 2016, 51, 3675--3683</jnl >.

$<$ lit46><jnl>L. Baptista-Pires, C. Mayorga-Martinez, M. Medina-Sanchez, H. Monton, A. Merkoci, ACS Nano 2016, 10, 853--860</jnl>.

Manuscript received: March 3, 2018

Revised manuscript received: April 5, 2018

Version of record online: $\langle$ ? $\rangle\langle$ ? $\rangle$

Figure $^{\wedge \wedge} 1$ Investigation of the morphology of graphene used: a) ${ }^{\wedge \wedge} \mathrm{TEM}$ image of the graphene sheets. $b)^{\wedge \wedge}$ SAED pattern. $\left.c, \wedge d\right)^{\wedge \wedge}$ SEM images of cross-section and top view of graphene print. e) $)^{\wedge \wedge}$ Raman spectra of the graphene sheets. f)^^Corresponding XRD pattern.

Figure $\left.{ }^{\wedge} 2 \quad a--c\right)^{\wedge \wedge}$ Graphene printed on both sides of the flexible paper. $\left.d\right)^{\wedge \wedge}$ Schematic diagram of the device assembly. Inset shows an assembled device used for electrochemical measurements.

Figure ${ }^{\wedge} 3$ a) ${ }^{\wedge \wedge}$ Cyclic voltammogram of the graphene device in conventional $\mathrm{H}_{2} \mathrm{SO}_{4}$ electrolyte. $b)^{\wedge \wedge}$ Charge--discharge curves.

Figure ${ }^{\wedge \wedge} 4 \quad$ a) $)^{\wedge}$ XPS spectra of graphene with KI adsorbed before and after electrochemical measurements. b)^^High-resolution magnification of the $I^{\wedge} 3 d$ signals. 
Figure ${ }^{\wedge \wedge} \quad$ a $)^{\wedge \wedge} \mathrm{CV}$ at $10^{\wedge \wedge} \mathrm{mV}^{\wedge} \mathrm{s}^{<\mathrm{M}->1}$ and $\left.\mathrm{b}\right)^{\wedge \wedge}$ charge--discharge curves at $6.5^{\wedge \wedge} \mathrm{mA}^{\wedge} \mathrm{cm}^{<\mathrm{M}->3}$ of the graphene device with different concentrations of KI.

c)^^Comparative volumetric capacitance with unmodified graphene and graphene with different KI concentrations at different current values. d)^^ Ragone plot of the device.

Figure ${ }^{\wedge} 6$ Electrochemical Impedance measurements: a)^^Nyquist Plot of the device with and enlarged view (inset). b)^^Bode plot of the micro-supercapacitor. $c)^{\wedge \wedge}$ Real and imaginary capacitances $\left(C^{\prime}\right.$ and $\left.C^{\prime \prime}\right)$ vs. frequency $\left(\log ^{\wedge} f\right)$ of graphene- and graphene+KIbased micro-supercapacitors in $1^{\wedge} \mathrm{M} \mathrm{H}_{2} \mathrm{SO}_{4}$.

Figure $^{\wedge \wedge}$ Capacitance retention of printed graphene device with and without KI species over 3000 charge--discharge cycles.

1. Cai, X., et al., Ultra High Electrical Performance of Nano Nickel Oxide and Polyaniline Composite Materials. Polymers, 2017. 9(7): p. 288.

2. Reber, D., R.-S. Kuhnel, and C. Battaglia, High-voltage aqueous supercapacitors based on NaTFSI. Sustainable Energy \& Fuels, 2017. 1(10): p. 2155-2161.

3. Xu, D., et al., Redox additives of Na2MoO4 and KI: Synergistic effect and the improved capacitive performances for carbon-based supercapacitors. Journal of Power Sources, 2017. 341: p. 448-456.

4. Zhang, Y., et al., An ultrahigh performance supercapacitors based on simultaneous redox in both electrode and electrolyte. Vol. 694. 2017.

5. Liu, Y., et al., Migration Chemistry and Behaviour of lodine Relevant to Geological Disposal of Radioactive Wastes: A Literature Review with a Compilation of Sorption Data. 1988: Nagra.

6. Spence, R.D., Chemistry and Microstructure of Solidified Waste Forms. 1992: Taylor \& Francis. 